Mens

revue d'histoire intellectuelle de l'Amérique française

\title{
Un médecin et son évêque : un incident dans l'histoire de la science et de la religion au Québec
}

\section{Ramsay Cook}

Volume 1, numéro 2, printemps 2001

URI : https://id.erudit.org/iderudit/1024445ar

DOI : https://doi.org/10.7202/1024445ar

Aller au sommaire du numéro

Éditeur(s)

Centre de recherche en civilisation canadienne-française

ISSN

1492-8647 (imprimé)

1927-9299 (numérique)

Découvrir la revue

Citer cet article

Cook, R. (2001). Un médecin et son évêque : un incident dans l'histoire de la science et de la religion au Québec. Mens, 1(2), 97-113.

https://doi.org/10.7202/1024445ar
Résumé de l'article

En 1911, Albert Laurendeau (1857-1920), un médecin de Saint-Gabriel-de-Brandon, publia, à compte d'auteur, un livre intitulé La vie : considérations biologiques, dans lequel il défendait une théorie lamarckienne de révolution et dénonçait l'absence des théories scientifiques modernes dans l'enseignement des sciences au Canada français. Avant la publication de cet ouvrage, il était déjà entré en conflit avec $\mathrm{M}^{\mathrm{gr}}$ Joseph-Alfred Archambault (1859-1913), évêque de Joliette, à cause de ses idées jugées modernistes : Laurendeau tenait à séparer la science et la religion. Ainsi, ce dernier avait peut-être anticipé la réaction de l'évêque, qui condamna le livre et réclama l'entière soumission de Laurendeau à l'autorité ecclésiastique. Laurendeau résista pendant quelques mois, mais finit par se soumettre à son évêque. Sept ans plus tard, après le décès de $\mathrm{M}^{\mathrm{gr}}$ Archambault, Laurendeau réitéra ses propos " scandaleux ", cette fois sans châtiment. Cet incident est important pour la compréhension des relations entre science et religion dans le Québec du début du $\mathrm{XX}^{\mathrm{e}}$ siècle. 


\title{
UNMÉDECIN ET SONÉVÊQUE: UN INCIDENT DANS L'HISTOIRE DE LA SCIENCE ET DE LA RELIGIONAU QUÉBEC ${ }^{1}$
}

\author{
RAMSAY COOK \\ Directeur général \\ Dictionnaire biographique du Canada \\ University of Toronto
}

\section{RÉSUMÉ}

En 1911, Albert Laurendeau (1857-1920), un médecin de Saint-Gabriel-deBrandon, publia, à compte d'auteur, un livre intitulé La vie : considérations biologiques, dans lequel il défendait une théorie lamarckienne de l'évolution et dénonçait l'absence des théories scientifiques modernes dans l'enseignement des sciences au Canada français. Avant la publication de cet ouvrage, il était déjà entré en conflit avec $\mathrm{M}^{\mathrm{gr}}$ Joseph-Alfred Archambault (1859-1913), évêque de Joliette, à cause de ses idées jugées modernistes : Laurendeau tenait à séparer la science et la religion. Ainsi, ce dernier avait peut-être anticipé la réaction de l'évêque, qui condamna le livre et réclama l'entière soumission de Laurendeau à l'autorité ecclésiastique. Laurendeau résista pendant quelques mois, mais finit par se soumettre à son évêque. Sept ans plus tard, après le décès de $\mathrm{M}^{\mathrm{g}}$ Archambault, Laurendeau réitéra ses propos "scandaleux», cette fois sans châtiment. Cet incident est important pour la compréhension des relations entre science et religion dans le Québec du début du XX' siècle.

\section{ABSTRACT}

In 1911 Albert Laurendeau (1857-1920), a medical doctor in Saint-Gabriel-de-Brandon, published a book entitled La vie : considérations biologiques in which he advanced and defended a Lamarckian version of evolution and criticized French Canadian educational institutions for failing to teach up-to-date scientific knowledge. Previous to the publication of this work, be had already come into conflict with Msgr Joseph-Alfred Archambault (1859-1913), the Bishop of Joliette, over his seemingly modernist attempt to separate science from religion and may even have anticipated the bishop's reaction to the book: condemnation and a demand for complete submission by the doctor. Laurendeau resisted for a few months, but then submitted. Seven years later, after the bishop's death, Laurendeau epeated bis views without penalty. This incident is significant for an understanding of the relationship between science and religion in early-twentieth-century Quebec. 
Vis-à-vis de Lamarck et de Darvin, nous sommes ici, dans notre province, au point où en était le monde chrétien au XIV'et au XV' siècles vis-à-vis Copernic et Galilée Albert Laurendeau, La vie : considérations biologiques,

Saint-Gabriel-de-Brandon, [s.é.], 1911, p. 109.

En janvier 1961, alors que le nouveau gouvernement libéral de Jean Lesage enclenchait un processus de modernisation de l'État québécois qu'on finirait par appeler la «Révolution tranquille», André Laurendeau (1912-1968) publiait un éditorial dans Le Devoir, où il déplorait l'influence persistante des idées scientifiques démodées au $\mathrm{Ca}$ nada français. Il s'était inspiré dans ses propos d'un récent discours du cardinal Paul-Émile Léger qui marquait, selon lui, une nouvelle attitude de l'Église à l'égard de la science, unettement en avance sur plusieurs secteurs de notre milieus. Dans son article, Laurendeau rendait également hommage à son oncle, le $D^{x}$ Albert Laurendeau, qui avait subi «une solide condamnation épiscopale» pour avoir soutenu la théorie selon laquelle l'homme descend du singe $e^{2}$.

Le directeur du Devoir fut sans doute surpris, quelques jours plus tard, lorsqu'il reçut un appel téléphonique de l'évêque de Joliette, $\mathrm{M}^{\mathrm{gr}}$ Édouard Jetté. Ce dernier tenait à lui expliquer que la condamnation épiscopale de 1912 ne faisait aucunement mention de «l'évolution» mais portait plutôt sur les «erreurs philosophiques et théologiques» de l'oncle Albert. André Laurendeau aurait alors remercié l'évêque pour sa mise au point, lui soulignant que «l'affaire singe» était bien enracinée dans la tradition orale de sa famille ${ }^{3}$. Aux yeux de Laurendeau, dont la foi était chancelante à l'époque, la mise au point de l'évêque avait probablement paru peu convaincante ${ }^{4}$. En effet, les précisions obscures de $\mathrm{M}^{\mathrm{gt}}$ Jetté dissimulaient mal la controverse qui avait entouré la carrière du $\mathrm{D}^{\mathrm{x}}$ Albert Laurendeau.

$* * *$

Albert était le fils aîné du $D^{\mathrm{r}}$ Joseph-Olivier Laurendeau, notable de Saint-Gabriel-de-Brandon, un village situé au nord-est de Joliette. Des sept enfants (cinq garçons et deux filles) de J.-O. Laurendeau, seul Albert, né en 1857, allait poursuivre une carrière en médecine. Les filles se marièrent, alors que Fortunat entra dans la Société de Jésus. J.-T.-R. devint financier et Clovis, marchand de meubles. Enfin, Arthur devint musicien et, éventuellement, maitre de chapelle de la cathédrale de Montréal. Albert Laurendeau fré- 
quenta d'abord l'école du village, puis entra en 1872 à l'École normale Jacques-Cartier de Montréal. Il étudia ensuite à l'École de médecine et de chirurgie de Montréal, alors affiliée au Victoria College de Cobourg, en Ontario. Dans l'ensemble, l'éducation que reçut le jeune Albert était assez inusitée pour un Canadien français catholique de l'époque. Il avait contourné deux des plus importants lieux de socialisation de l'élite canadienne-française : le collège classique et la très catholique Université Laval. D'ailleurs, son cheminement scolaire peu commun pourrait expliquer en partie ses opinions scientifiques et pédagogiques non orthodoxes. Diplômé en 1879, il épouse la même année Marie-Georgiana Mérizzi, fille d'un notaire de Napierville. Quatre enfants naîtront de cette union. Le garçon, Aldéric, opta lui aussi pour la médecine. Des trois filles, deux se marièrent, dont une avec le secrétaire particulier de sir William Van Horne ${ }^{5}$. La troisième demeura à la maison.

Une fois diplômé, Laurendeau se mit à pratiquer la médecine à Saint-Gabriel-de-Brandon avec son père, auquel il succéda graduellement. Il se tailla rapidement une solide réputation de médecin dévoué, de praticien énergique doté d'idées fermes sur les responsabilités associées à sa profession, sur le statut de celle-ci, de même que sur la nécessité de la réformer. Citoyen engagé, il fut maire de Saint-Gabrielde-Brandon de 1889 à 1892 . Par la suite, il se retira de la politique municipale pour se consacrer à la politique interne de la profession médicale. Peu après le premier Congrès des médecins de langue française de l'Amérique du Nord à Québec en 1902, Laurendeau fonda la Société médicale du district de Joliette, l'une des associations médicales les plus actives dans la province, dont il fut pendant longtemps le secrétaire-trésorier. De 1904 à 1920, il siégea presque sans interruption au Conseil d'administration du Collège des médecins et chirurgiens de la province de Québec, puis accéda à sa vice-présidence en $1918^{6}$. À titre de président du comité législatif du Collège, il exerça des pressions sur l'Assemblée législative en faveur de l'adoption d'une nouvelle loi médicale en 1909. Celle-ci augmentait l'autonomie du Collège et étendait ses attributions. De plus, elle standardisait les exigences d'admission, les examens et les programmes des facultés de médecine au Québec. La loi de 1909 eut un impact profond sur l'enseignement médical au Québec, même si la part du programme consacrée aux humanités classiques dans les facultés de médecine demeurait toujours plus grande que celle accordée à la science. Selon Laurendeau, le but de la nouvelle loi serait de «relever la qualité et abaisser la quantité des disciples d'Hippocrate dans notre province ${ }^{7} \gg$. Bien qu'il ait souhaité une collaboration étroite entre les associations provinciales de médecins du Canada et l'établissement d'un ministère fédéral 
de la Santé ${ }^{8}$, le $D^{x}$ Laurendeau insistait pour que les associations médicales provinciales conservent leur autonomie. En effet, avec une association autonome, les médecins canadiens-français du Québec pourraient "garder intacts notre langue, nos usages et coutumes, notre organisation nationale, nos droits en un $\operatorname{mot}^{2} \%$.

Afin d'accroître, par une réforme, le degré de professionnalisation de la médecine québécoise, Laurendeau souhaitait renforcer la déontologie médicale, exclure les ramancheurs, guérisseurs et autres "charlatans» de la pratique, mieux réglementer l'incurie professionnelle par le biais du Collège des médecins et exercer un meilleur contrôle sur les médicaments brevetés. Pour Laurendeau, deux problèmes généraux devaient être réglés afin de hausser les exigences de la pratique médicale et le statut des médecins du Québec: d'une part, l'engorgement de la profession et d'autre part, la «formation morale, intellectuelle et scientifique» inadéquate des médecins canadiens-français. Pour Laurendeau, l'engorgement était devenu si sévère que de nombreux médecins se voyaient obligés d'avoir un emploi supplémentaire pour subvenir aux besoins de leur famille. D'ailleurs, il était de ce nombre. À divers moments de sa vie, comme d'autres médecins de la province de Québec, il fit des affaires, en l'occurrence, des opérations bancaires d'escompte et la promotion de la fabrication de fibre de bois et de potasse. La situation de Saint-Gabriel-de-Brandon, où «deux se font une rude concurrence dans le commerce de pharmacie et [où] moimême pendant longtemps, $j$ 'ai tenu un comptoir d'escompte ${ }^{10}$, , n'était pas exceptionnelle. Une concurrence féroce et des honoraires insuffisants obligeaient Laurendeau à mener ce qu'il appelait, à l'instar de Darwin, une «lutte pour la vie». Une solution au problème, adoptée par la Société médicale du district de Joliette en 1913, était l'uniformisation des honoraires : 1 ou $2 \$$ pour une visite à domicile, de 5 à $10 \$$ pour la réduction d'une fracture simple, de 40 à $60 \$$ pour une appendicectomie. Avec l'augmentation des revenus viendrait le prestige. Pour Laurendeau, «le médecin doit occuper une position élevée dans la hiérarchie sociale, et il ne peut tenir son rang, commander la confiance, le respect du public, sans avoir des revenus convenables ${ }^{11} \gg$.

La hausse des honoraires n'était qu'une des propositions du docteur pour amener une transformation profonde de la profession médicale au Québec. Une réforme pédagogique qui hausserait les exigences et abaisserait le nombre de diplômés était tout aussi importante. La loi de 1909 avait haussé les exigences de certification, mais il fallait réformer davantage le réseau québécois de l'enseignement. Laurendeau jugeait nécessaire une réorientation radicale. À son 
avis, l'ensemble du réseau d'enseignement canadien-français avait pour principale faiblesse de compter trop de collèges classiques et trop peu de collèges techniques, agricoles, industriels et commerciaux. Le résultat de cette faiblesse sautait aux yeux : dans une société où les uétrangers» dominaient l'économie, les collèges classiques produisaient un surplus de prêtres et de membres des professions libérales ${ }^{12}$. Plus grave encore, d'après Laurendeau, était le manque de respect pour la science que l'on retrouvait chez les Canadiens français. Cette carence était également le résultat d'un système d'enseignement mal adapté aux besoins d'une société moderne. Le programme classique privilégiait les langues mortes aux dépens des langues vivantes. Selon Laurendeau, les collèges classiques devaient plutôt accorder une priorité à l'enseignement de l'anglais, au détriment du grec et du latin. Dans son esprit, la langue anglaise "pourrait être le levier de notre succès». La langue française, pour sa part, conserverait sa primauté «à cause de sa supériorité sur les autres idiomes». Pour les étudiants se dirigeant vers la médecine, Laurendeau voulait remplacer la philosophie classique par un nouveau système qui «enseignerait à nos jeunes gens les principes généraux des sciences positives et naturelles - en s'appuyant uniquement sur les procédés et les faits naturels guidés seulement par la raison ${ }^{13}$ ).

$\mathrm{Au}$ cours de la décennie précédant la Première Guerre mondiale, Laurendeau acquit une position enviable, voire même une certaine notoriété, au sein de la profession médicale québécoise, à cause de ses conférences données pour des organisations professionnelles et de ses contributions fréquentes à des revues médicales telles L'Union médicale $d u$ Canada et La Clinique. Son association avec cette dernière revue est particulièrement intéressante. En effet, La Clinique avait été fondée par le $\mathrm{D}^{\mathrm{r}}$ François-Xavier Le Moyne de Martigny qui, comme son frère Adelstan, également médecin, était un franc-maçon notoire. Or, on sait que la franc-maçonnerie était une organisation anticléricale secrète étroitement surveillée par le clergé. Laurendeau devait bien connaître le fondateur de La Clinique, puisqu'ils avaient siégé ensemble au comité du Collège des médecins et chirurgiens chargé de la réforme de la loi médicale du Québec. Bien que Laurendeau, semble-t-il, ne soit jamais devenu membre de la franc-maçonnerie, sa vision de l'éducation et de la science était souvent partagée par des membres des redoutées loges maçonniques. Au Canada français de l'époque, cette association était suffisante pour suggérer la culpabilité du $D^{x}$ Laurendeau ${ }^{14}$.

Les conceptions scientifiques de Laurendeau s'étaient formées lentement par des lectures très variées sur les sciences naturelles de la fin du $\mathrm{XIX}^{\mathrm{c}}$ siècle et étaient fondées sur une connaissance profonde du 
débat scientifique et religieux entourant la théorie de l'évolution. Au Québec, où sir William Dawson (1820-1899), directeur presbytérien de l'Université McGill, l'abbé Léon Provancher (1820-1892) et d'autres savants catholiques avaient dénoncé le darwinisme, Laurendeau courait d'énormes risques à discuter ouvertement des mérites de la théorie de l'évolution. En mars 1907, il déclencha une très longue controverse en exposant de façon officieuse la doctrine évolutionniste moderne à ses collègues de la Société médicale du district de Joliette. $\mathrm{M}^{\mathrm{gr}} \mathrm{Joseph}$ Alfred Archambault (1859-1913), nommé premier évêque de Joliette en 1904 et ex-vice-recteur de la succursale montréalaise de l'Université Laval, réagit sans délai. Il exigea des explications complètes, incluant une preuve de la soumission du docteur «à l'autorité suprême et infaillible de l'Église». Laurendeau répondit en soulignant qu'il n'avait pas exposé le darwinisme en tant que dogme, mais plutôt en tant que théorie si largement acceptée par les biologistes, que les hommes instruits, en particulier les médecins, devaient connaître ses préceptes. Il se défendit d'avoir une quelconque hostilité envers la religion et nia l'existence d'un conflit entre le Dieu de la Genèse et la science moderne. Pour Laurendeau, le débat entourant l'évolutionnisme ne devait pas être la chasse gardée des libres-penseurs ${ }^{15}$. Plutôt que de se rétracter devant son redoutable adversaire, Laurendeau décida de préparer une présentation plus officielle de son point de vue et de solliciter la participation de $\mathrm{M}^{\mathrm{gr}}$ Archambault à une discussion publique où l'évêque pourrait "exposer les doctrines chrétiennes sur ces questions ${ }^{16}$ ".

Au cours de la discussion, où l'évêque brillait par son absence, Laurendeau fit appel à l'autorité de nombreux auteurs catholiques européens, tels le Cardinal-Archevêque de Paris, $\mathrm{M}^{\mathrm{gr}}$ Joseph-Hippolyte Guibert (1802-1886), ou le professeur anglais St. George Jackson Mivart (1827-1900), qui prétendaient que la religion et l'évolution n'étaient pas en conflit ${ }^{17}$. En dépit des propos plutôt modérés du docteur, l'un de ses collègues de la Société médicale, le $\mathrm{D}^{\mathrm{x}}$ Bartolet, prit la parole pour dénoncer son «matérialisme». À cela, Laurendeau répliqua que «l'homme, son origine et sa fin matérielles, sont strictement du domaine de la médecine ${ }^{18}$ ). Selon Laurendeau, les questions de l'existence de Dieu et de l'immortalité de l'âme dépendaient d'un autre ordre, séparé de celui de la science. Il croyait fermement en la nécessité d'établir une distinction entre science et religion, position dans laquelle l'évêque reconnut l'hérésie du modernisme. En effet, le modernisme, mouvement ayant particulièrement affecté le catholicisme français et italien sous le pontificat de Pie X (1903-1914), incluait entre autres la séparation de la religion et de la science ${ }^{19}$. Implicitement, les modernistes reconnaissaient l'existence d'une sphère de pensée laïque autonome par rapport au sacréé $^{20}$. 
Pour Laurendeau, «'humanité ne peut rien perdre, elle ne peut que gagner, au point de vue intellectuel et moral, par la diffusion de la science et de la vérité21 $\gg$. Par ailleurs, il se rendait encore plus condamnable aux yeux de son évêque en citant Haeckel, Voltaire, Kant et Lacordaire, de même qu'en s'attaquant à̀l'Inquisition!

Aux yeux de $\mathrm{M}^{\mathrm{gr}}$ Archambault la «vérité» était tout autre. Réclamant de nouveau la soumission du médecin à l'autorité et aux doctrines de l'Église, l'évêque l'invita à se rendre à la cathédrale de Joliette le dimanche suivant, où il expliquerait en chaire les «erreurs du modernisme ${ }^{23}$ \%. De nouveau, Laurendeau nia que sa défense de l'évolutionnisme contrevenait aux enseignements catholiques («le catholicisme peut admirablement se concilier avec la vraie science») et son adhésion à n'importe laquelle des soixante-cinq propositions condamnées par le pape $\mathrm{Pie} X$ dans son syllabus antimoderniste, le décret Lamentabili sane exitu (1907). Il disait accepter l'infaillibilité de l'Église dans le domaine de la foi et de la moralité, tout en réaffirmant son attachement à la vérité scientifique de l'évolutionnisme ${ }^{23}$.

Cette mise au point était cependant loin de satisfaire $\mathrm{M}^{\mathrm{gx}}$ Archambault. Celui-ci tenait à ce que la soumission du docteur ne comporte aucune réserve. L'évêque insista pour que Laurendeau accepte six propositions théologiques : cinq portaient sur la primauté du spirituel sur le matériel, et la sixième était que la vérité scientifique ne pouvait en aucun cas contredire la vérité religieuse telle que proclamée par l'Église. Il ne pouvait donc être question de séparation de la science et de la religion. Lorsque Laurendeau se mit à temporiser, $\mathrm{M}^{\mathrm{g}}$ Archambault lui donna huit jours pour choisir entre la soumission et la condamnation. Deux jours plus tard, le docteur se soumit. Toutefois, il n'avait pas changé d'avis. En effet, pour Laurendeau, l'époque du géologue et paléontologiste suisse Louis Agassiz (1807-1873) et du naturaliste et anthropologue français Jean-Louis-Armand de Quatrefages de Bréau (1810-1892) était révolue ${ }^{24}$. Depuis, aucun savant sérieux ne pouvait défendre la fixité des espèces ou prétendre que la terre n'avait été créée qu'en 4004 avant Jésus-Christ. Laurendeau écrivit donc à son évêque pour lui rappeler que "par deux fois, publiquement, $j$ 'ai répété que les conflits entre la science et la foi, proviennent non de la science ni de la religion, mais des savants qui interprètent mal les faits, ou des théologiens qui ne pénètrent pas le sens des Écritures». $\mathrm{M}^{\mathrm{g}}$ Archambault refusa de relancer la polémique. Il accepta la soumission du docteur avec «la joie dans l'âme» et déclara que «l'incident est $\operatorname{clos}^{25}$ ». L'évêque avait tort. Laurendeau ne s'était pas vraiment soumis, il avait signé une trêve. D'ailleurs, ses convictions scientifiques 
s'affermirent dans les années suivantes, de sorte que la trêve ne dura pas.

En juillet 1908 à Québec, Laurendeau utilisa son poste de président de la troisième section du Congrès des médecins de langue française de l'Amérique du Nord pour répéter, sans mettre de gants blancs, sa critique radicale du système d'enseignement provincial. Dans un discours prononcé au Congrès, il s'attaqua en particulier au caractère arriéré de l'enseignement scientifique canadien-français dont le résultat était, selon lui, une profession médicale conservatrice, voire même réactionnaire. Dans son esprit, le contrôle externe, c'est-à-dire clérical, de l'embauche dans les facultés de médecine nuisait aux standards de la profession. Le problème, selon Laurendeau, était philosophique, car l'idéalisme dominait encore l'empirisme dans l'enseignement des sciences et de la médecine. Pour lui, «la philosophie doit avoir pour base la raison fondée sur nos sensations; hors de là, nous tomberons dans la métaphysique, le mysticisme, le transcendant, toutes choses que doit repousser le médecin, lequel doit étudier et traiter l'homme et ses maladies, par les procédés naturels $\left.{ }^{26}\right\rangle$. Le vrai raisonnement scientifique nécessitait le rejet de la loi naturelle et, implicitement, du créationnisme chrétien. «La croyance à la fixité des espèces conduit à l'inertie intellectuelle [et] la foi en une essence humaine physique, différentielle des autres animaux, mène à l'anthropolâtrie : dogmes opposés à la raison et antiscientifiques ${ }^{27}$.» Après quelques réflexions sur la nécessité d'améliorer les lois sanitaires de la province (il tenait à ce que les «temples religieux» soient inclus dans l'application des lois) et de bien isoler les tuberculeux, sur la prohibition, de même que sur la mise en place d'un plan d'action pour combattre la prostitution afin d'entraver la propagation de la syphilis, il conclut son discours en affirmant que la science était le seul moyen fiable pour atteindre le progrès social et moral ${ }^{28}$.

Laurendeau était fier d'avoir et d'exprimer des idées qu'il tenait pour «avancées». Toutefois, ses collègues étaient loin de partager ses idées ou son enthousiasme. «Le sentiment général de l'assemblée», pouvait-on lire dans L'Union médicale du Canada après le discours du docteur, «ne lui a pas permis de terminer l'exposé de son travail ${ }^{29}$ 1). Cela ne l'empêcha toutefois pas de faire imprimer et distribuer le texte de son discours. Quand cette nouvelle déclaration en faveur du naturalisme scientifique fut portée à l'attention de $M^{8 \mathrm{r}}$ Archambault à l'automne 1910, il la qualifia de «scandale». Furieux, l'évêque réclama une rétractation ou, au minimum, que Laurendeau permette la publication de la correspondance de 1907 , incluant sa soumis- 
sion à l'autorité de l'Église. Initialement, Laurendeau acquiesça à la publication de sa correspondance, mais finit par offrir «une rétractation directe» de tout ce qui pourrait contredire les enseignements de l'Église à son évêque ${ }^{30}$. De nouveau, Laurendeau se soumit à $\mathrm{M}^{\mathrm{gr}}$ Archambault. Mais encore une fois, le débat était loin d'être réglé.

En fait, il est probable qu'à l'époque de sa deuxième soumission à l'évêque, c'est-à-dire en octobre 1910, Laurendeau planifiait déjà la publication de son livre intitulé La vie : considérations biologiques, qui parut au début de 1911. Le docteur savait sûrement que son livre, qui contenait sa correspondance avec $\mathrm{M}^{\mathrm{gr}}$ Archambault et qui ne portait ni l'imprimatur ecclésiastique, ni la marque d'un imprimeur, relancerait la controverse. En effet, il avait fait publier La vie : considérations biologiques précisément pour «briser des vieilles traditions, secouer d'antiques préjugés, et par-dessus tout, troubler la quiétude de la masse de la hiérarchie ${ }^{31}$ ). Laurendeau plaida pour que son ouvrage soit jugé à l'aune de standards scientifiques et non religieux, insistant de nouveau sur l'existence autonome de la science. Toutefois, il savait sans doute que cet appel à l'empirisme était futile puisque dans son livre, il affichait explicitement et sans réserve son adhésion au naturalisme scientifique, une doctrine qui cherchait à expliquer la vie par une combinaison des théories de Charles Darwin (1809-1882) et de Jean-Baptiste de Monet, chevalier de Lamarck (1744-1829). Attaquant le créationnisme «puérill» enseigné dans les collèges classiques du Québec, Laurendeau affirmait que «la vérité réside dans l'évolution de Lamarck, de Darwin et de la pléiade de savants qui ont assis cette doctrine sur des bases solides ${ }^{32}$ ?. La théorie de l'évolution ou le «transformisme», expression lamarckienne préférée par le docteur, s'appliquait autant à l'homme qu'à la nature. Selon lui, seul le transformisme pouvait expliquer les différences entre les races supérieures et inférieures ou la quasi-disparition de peuples tels les Hurons, les Iroquois ou les Abénakis $^{33}$.

Malgré les lectures étendues de Laurendeau sur l'évolution, dont la Pbilosophie zoologique (1809) de Lamarck, il est peu probable qu'il ait lu On the Origin of Species (1859) de Darwin. Et même si le docteur avait lu Darwin, il n'en demeure pas moins qu'il n'a pas réussi à cerner la différence essentielle entre la vision lamarckienne d'une évolution verticale, incluant une réponse aux défis de l'environnement et la transmission héréditaire des caractéristiques acquises, et la théorie darwiniste d'une évolution bifurquant par sélection naturelle. Laurendeau, au contraire de Darwin, mais à l'instar de Lamarck, croyait que l'évolution était progressive ${ }^{34}$. Sa pensée avait subi l'influence des néolamarckiens français qui, pour des raisons linguistiques, idéologiques et 
nationalistes, insistaient sur le fait que l'œuvre de Charles Darwin complétait, sans les détrôner, les théories évolutionnistes antérieures de Lamarck. De plus, la prudence darwiniste avait peu d'attrait pour le fougueux $\mathrm{D}^{\mathrm{r}}$ Laurendeau ${ }^{35}$.

Après avoir expliqué sa vision de l'évolution, le polémiste de Saint-Gabriel-de-Brandon présenta les conséquences pratiques de ses idées. Pour lui, la science était la clef de l'avenir, mais une clef refusée aux Canadiens français. «Au XVIII ${ }^{e}$ siècle, il a fallu une langue philosophique, au XIX $\mathrm{XIècle}^{\mathrm{e}}$ une langue poétique, au XX $\mathrm{XX}^{\mathrm{e}}$ siècle il faudra une langue scientifique ${ }^{36} . »$ La connaissance scientifique, c'est-à-dire l'évolutionnisme, lui apparaissait nécessaire pour garantir une science solide et expliquer une évolution morale, intellectuelle et sociale qui ferait nécessairement en sorte que «l'utopie d'aujourd'hui deviendra la réalité de demain ${ }^{37}$ ». Pour Laurendeau, aucun peuple n'avait un plus grand besoin de la science que le Canada français. L'avenir des Canadiens français serait sombre à moins qu'ils n'arrivent à mettre de côté leurs croyances et leur philosophie démodées au profit des ugrandes lois de l'évolution 38 i).

Laurendeau pouvait s'attendre à une réaction rapide de $\mathrm{M}^{\mathrm{gr}}$ Archambault. En effet, l'évêque semblait porter beaucoup d'attention aux faits et gestes du docteur ${ }^{39}$. Le 19 mars 1912, Mgr Archambault émit une circulaire condamnant $L a$ vie et interdisant aux laïcs de le lire. Dix jours plus tard, le message fut reproduit dans L'Étoile du Nord. Dans une prose vigoureuse et sans ambiguité, l'évêque contreattaquait :

J'y ai constaté des erreurs assez nombreuses en matière de philosophie chrétienne et de théologie catholique; en outre, des injures toutes gratuites à l'adresse de la hiérarchie, de notre clergé canadien-français, des professeurs de nos universités et de nos collèges, des éloges exagérés des savants et des philosophes athées et matérialistes, des insinuations regrettables au sujet des guérisons miraculeuses, de la confiance des fidèles dans le pouvoir d'intercession des saints, un mépris évident de l'enseignement scolastique, etc. Parmi les graves erreurs doctrinales, que contient le livre La vie, il y en a même plusieurs au sujet de la liberté humaine, de la nature de notre intelligence, de l'autorité de l'Église en matière scientifique, des relations de la science et de la foi, de la puissance de la raison humaine de connaître l'existence de Dieu et l'immortalité de l'âme; erreurs qui ont été formellement condamnées soit par le Concile du Vatican, soit par le décret Lamentabili, et par l'encyclique Pascendi ${ }^{40}$. 
Toutefois, $\mathrm{M}^{\mathrm{gx}}$ Archambault ne fit aucune référence explicite aux idées évolutionnistes de Laurendeau. En effet, un principe plus fondamental pour l'évêque était en jeu, celui de la primauté de l'autorité religieuse.

Sommé de se rétracter, Laurendeau souligna de nouveau qu'il n'était pas hostile à la religion, tout en réclamant le droit d'exprimer ses idées. Il réaffirma son intention d'étudier les grandes questions scientifiques, et de ne s'exprimer publiquement que si on le provoquait. De nouveau, il nia que la science ait une autorité quelconque sur la croyance ou les enseignements religieux. Dans une lettre à son évêque, Laurendeau tenait à clore ce nouvel incident :

À la suite de ces déclarations de principes, il vous appartient, Monseigneur, de juger si je puis encore faire partie de la société religieuse, ou si je dois en être définitivement et pour toujours exclu. Toute autre déclaration me déshonorerait à mes yeux; je croirais commettre une infamie en affirmant ce que ma conscience répudie. Je puis vous affirmer que mes convictions sont aussi profondes et sincères que les vôtres. Vous êtes maintenant juge et je m'en rapporte à vous ${ }^{41}$.

$\mathrm{M}^{\mathrm{g}}$ Archambault était prêt à juger. Laurendeau devait se soumettre à l'évêque par écrit ou se voir interdire les sacrements, y compris une sépulture catholique ${ }^{42}$.

Pendant neuf mois, le docteur tint bon. Faute de sources, nous ne savons pas comment cette intransigeance fut accueillie par sa famille, en particulier par son frère Fortunat, le Jésuite, et par ses amis. Néanmoins, la pression exercée sur le $D^{r}$ Laurendeau devait être intense. En effet, sa pratique médicale et sa subsistance étaient menacées par son obstination. Le 4 mars 1913, il visita $\mathrm{M}^{\mathrm{gr}}$ Archambault et le lendemain, admit ses erreurs :

J'accepte l'enseignement de l'Église catholique, je regrette les erreurs que ce livre peut contenir; au point de vue de la doctrine chrétienne, les erreurs proviennent sans doute de ce que mon instruction religieuse est insuffisante; aussi, désirant la paix, ayant donné à mon activité un but autre que celui d'atteindre aux sommets scientifiques - $j$ 'ai résolu de ne plus écrire $e^{43}$.

Laurendeau demanda à l'évêque de considérer l'incident comme étant clos. Il espérait que sa décision ne serait pas rendue publique. Néanmoins, le lendemain, le «Bulletin religieux» de L'Étoile $d u$ Nord annonçait sa «soumission à l'autorité ecclésiastique» et la 
rétractation de toutes les erreurs doctrinales contenues dans La vie ${ }^{44}$. Pour $\mathrm{M}^{\mathrm{gr}}$ Archambault, cette notice publique discrète était une part nécessaire de l'entente. Il ira même jusqu'à féliciter son adversaire défait pour «avoir, sous l'action de la grâce divine, accompli son devoir ${ }^{45} \%$.

Une fois de plus, l'incident était clos : les habitants de Joliette étaient à l'abri du naturalisme scientifique et le docteur, réduit au silence. Laurendeau reprit sa routine habituelle. Il visitait ses patients, enseignait l'hygiène dans les écoles locales, prêchait la tempérance et continuait à être réélu à divers postes dans les associations médicales du Québec. De nouveau, Laurendeau fut accepté par ses collègues, qui admiraient son énergie et son intelligence, même s'ils étaient souvent en désaccord avec ses idées radicales. Laurendeau consacrait une partie de son temps à des entreprises de fabrication de fibre de bois et de potasse qui firent faillite durant la Première Guerre mondiale. Apparemment, il mit au point et fit breveter avec succès au moins un instrument chirurgical amélioré ${ }^{46}$. Peu après sa soumission, il prit sans aucun doute connaissance de la mort subite de $\mathrm{M}^{\mathrm{gr}}$ Archambault, le 25 avril $1913^{47}$.

$\mathrm{Au}$ cours des années suivantes, moins mouvementées, Laurendeau continua à méditer ses convictions évolutionnistes et son humiliation aux mains de $\mathrm{M}^{\mathrm{g}}$ Archambault. Suite au décès de l'évêque, il a peut-être cherché une nouvelle occasion de soulever la controverse. À la fin de 1918, cette occasion survint presque par accident. Laurendeau fut invité à se joindre au comité éditorial de La Clinique, autre signe de l'estime dont il jouissait toujours auprès de ses collègues. Toutefois, il déclina cette offre en affirmant de façon badine qu'il était né dans les bois et demeurait non civilisé. Rappelant brièvement ses convictions évolutionnistes, il affirma que ubref, je pourrais être un trouble-fête, comme je l'ai été déjà ${ }^{48}$ \%. Le mois suivant, il exposa ses idées de façon plus détaillée, en soutenant que le rédacteur en chef de la revue les avait mal présentées. Le jour de l'armistice, le 11 novembre 1918, Laurendeau fit éclater une nouvelle bombe intellectuelle. Dans les pages de La Clinique, il tenta d'expliquer le sens de son livre condamné, La vie : considérations biologiques. Clairement impénitent, il prétendit que $\mathrm{M}^{\mathrm{gr}}$ Archambault l'avait menacé d'excommunication sans lui avoir donné la possibilité de s'expliquer. De nouveau, il réitéra sa foi en la théorie de l'évolution. De plus, il affirma que sans liberté de pensée, la véritable science ne pourrait s'épanouir dans les universités du Canada français. Le temps était venu, selon lui, de nommer non seulement des catholiques, mais aussi des libres-penseurs, 
des protestants et des Juifs aux postes universitaires, et de leur accorder l'autonomie dans leur enseignement. Pour lui, la tolérance était la «mère de la concorde et du progrès $\left.{ }^{49}\right)$. Au fond, le temps avait renforcé le libéralisme de Laurendeau. Une brève controverse suivit ces propos. Celle-ci permit au docteur de conclure qu'au Québec, «nous en sommes encore à l'âge médiéval ${ }^{50} \%$. Toutefois, l'incident fut de courte durée. Apparemment, les autorités ecclésiastiques préféraient le silence à une autre controverse.

Une fois la soixantaine entamée, Laurendeau continua à pratiquer la médecine, à écrire et à s'adonner à la politique de sa profession. À la fin de la guerre, Joliette, comme ailleurs au Canada, fut frappée par l'épidémie de grippe espagnole. Laurendeau travailla d'arrache-pied pour combattre le fléau et, comme par le passé, rendait compte de ses traitements parfois novateurs à ses collègues. Il s'enorgueillirait, avec raison, de n'avoir perdu que 12 des quelque $500 \mathrm{mala}-$ des qu'il traita dans sa région ${ }^{51}$, dont environ 300 à Saint-Gabriel-deBrandon seulement. Ce surmenage, ou une vie marquée par l'action, la tension et la controverse, furent peut-être à la source de la crise cardiaque qui l'emporta, le 19 août 1920. La semaine précédente, comme d'habitude, avait été remplie de réunions professionnelles. Pratiquant la médecine jusqu'au jour de sa mort, il rendit l'âme au volant de son automobile, près de son bureau à Saint-Gabriel-de-Brandon, après avoir visité un patient. Le 23 août, ses funérailles furent célébrées par son frère, Fortunat Laurendeau, s.j., à l'église paroissiale ${ }^{52}$. Le sort du $\mathrm{D}^{\mathrm{r}}$ Albert Laurendeau ne serait donc pas celui de Joseph Guibord (18281869), qui repose toujours dans une tombe désacralisée du cimetière de la Côte-des-Neiges à Montréal ${ }^{53}$.

Résumant la vie tumultueuse du $\mathrm{D}^{\mathrm{r}}$ Laurendeau, son collègue, le $D^{r}$ Joseph Gauvreau, écrivit que son «originalité consistait surtout à n'être pas souvent de l'avis de tout le monde ${ }^{54} \gg$. De plus, la vie d'Albert Laurendeau nous offre un aperçu de la carrière d'un médecin de campagne et de la professionnalisation de la pratique médicale au Québec. Laurendeau était un chef de file de ce mouvement, même si ses appuis au sein de la profession étaient loin d'être unani$\mathrm{mes}^{55}$. La vie du docteur nous permet en outre de mieux comprendre l'état des idées scientifiques et religieuses dans la culture québécoise du début du $\mathrm{XX}^{\mathrm{e}}$ siècle, de même que la dimension canadienne-française de la lutte antimoderniste du pape Pie X. À l'époque, Laurendeau avait peu d'appuis dans sa croisade en faveur du progressisme scientifique. 
Le conflit entre le docteur et $\mathrm{M}^{\mathrm{gr}}$ Archambault illustre bien la détermination de l'Église québécoise à protéger ses ouailles contre les erreurs du modernisme, dont la plus pernicieuse était selon elle la séparation de la science et de la religion. La victoire initiale fut celle de l'évêque, mais, à la fin de la Première Guerre mondiale, les idées de Laurendeau semblaient tolérées, ou du moins ignorées. Le combat serait de longue durée. En dépit de la fixité apparente du Québec rural, de nouvelles idées et influences y circulaient. Lentement, elles transformeraient profondément la société canadienne-française. Le $\mathrm{D}^{\mathrm{r}}$ Albert Laurendeau avait ardemment souhaité cette modernisation. "Ce n'est pas une révolution que je prêche, écrira-t-il, c'est l'évolution de notre grande institution canadienne-française [1'Université Laval] dans son intérêt et dans l'intérêt de notre race ${ }^{56} . »$ Toutefois, comme le savait le $\mathrm{D}^{\mathrm{x}}$ Laurendeau, l'évolution est un lent processus ${ }^{57}$.

\section{Traduction : Damien-Claude Bélanger et Sophie Coupal ${ }^{58}$}

\section{NOTES}

' L'abbé François Lanoue nous a généreusement donné accès à la correspondance entre $\mathrm{M}^{\mathrm{g}} \mathrm{J}$ Joseph-Alfred Archambault et Albert Laurendeau, qui est conservée dans les Archives de l'évêché de Joliette. Nous tenons à le remercier, ainsi que Xavier Gélinas, qui a effectué pour nous des recherches indispensables dans les périodiques médicaux du Québec. Le professeur Yvan Lamonde et le regretté professeur Jean Hamelin ont eu la gentillesse de lire et de commenter une version anglaise de cette étude.

2 André Laurendeau, «Une réponse actuelle», Le Devoir, 31 janvier 1961, p. 4.

${ }^{3}$ Archives de l'évêché de Joliette (AÉJ), Extrait du livre de procies-verbal de la Société bistorique de Joliette, Volume II : 1947-1968, pp. 125-126.

4 Yves Laurendeau, «En guise de supplément au Laurendeau de Monière», Revue d'histoire de l'Amérique franfaise, vol. 38, $\mathrm{n}^{\circ} 1$ (été 1984), p. 76. La décision qu'aurait prise André Laurendeau de garder confidentielle la remise en question de sa foi pourrait être attribuée à la tradition familiale entourant le destin de son oncle Albert. ${ }^{5}$ [S.a.], «Le docteur Albert Laurendeau décédé», L'Étoile du Nord, 26 août 1920, p. 1; «Mémoire concernant l'enseignement secondaire canadien-français, lu à l'assemblée des gouverneurs du Collège des médecins et chirurgiens de la province de Québec par le docteur Albert Laurendeaus, La Clinique, vol. VIII, $n^{\circ} 5$ (août 1917), p. 157; au sujet de l'École normale Jacques-Cartier, voir : Thérèse Hamel, «Verreau, Hospice-AnthelmeJean-Baptiste", dans Ramsay Cook, dir. Dictionary of Canadian Biography, Volume XIII, Toronto, University of Toronto Press, 1994, pp. 1049-1053; au sujet de l'École de médecine, voir : Denis Goulet, Histoire de la Faculté de médecine de l'Université de Montréal, 1843-1993, Montréal, VLB, 1994, p. 40-49.

'Joseph Gayvreau, «Nécrologie: Albert Laurendeau, 1857-1920), LUnion médicale du Canada, vol. 49, n० 9 (septembre 1920), p. 463.

7 [Albert Laurendeau], «Intérêt professionneb), La Clinique, vol. I, no 1 (avril 1910), p. 4; «Intérêts professionnels. Nos sociétés médicales. La loi médicale», La Clinique, vol. I, $n^{\circ} 10$ (janvier 1911), p. 331. Au sujet de la législation de 1909, voir : Goulet, op. cit., pp. 128-129; Denis Goulet et André Paradis, Trois siècles d'histoire médicale an Québec. 
Chronologie des institutions et des pratiques (1639-1939), Montréal, VLB, 1992, pp. 368369 et Statutes of the Province of Quebec (1909), chapitre 55.

${ }^{8}$ Albert Laurendeau «Association médico-chirurgicale du district de Joliette», LUnion médicale du Canada, vol. XLIII, $\mathrm{n}^{\circ} 10$ (octobre 1914), p. 438.

'Idem, «Bill Roddick», La Clinique, vol. I, n² 2 (mai 1910), p. 38.

${ }^{10}$ Idem, "Intérêts professionnels. Nos sociétés...., loc. cit., p. 329.

"Idem, "Un exemple à suivre», La Clinique, vol. V, no 1 (avril 1914), pp. 5-9, 10.

12 Idem, "Intérêts professionnels. Nos sociétés...", loc. cit., p. 331.

${ }^{13}$ Idem, "Mémoire concernant....), loc. cit., pp. 159-160.

${ }^{14}$ Roger Le Moine, «Le Grand Orient de France dans le contexte québécois (18961923)", dans Yvan Lamonde, dir. Combats libéraux au tourmant du XX' siècle, Montréal, Fides, 1995, p. 154; Roger Le Moine, Deux loges montréalaises du Grand Orient de France, Ottawa, PUO, 1991, p. 130; [Albert Laurendeau], «Intérêt professionneb), La Clinique, vol. I, n 1 (avril 1910), p. 1. A.-J. Lemieux, La loge L'Émancipation, Montréal, La Croix, 1910. Nous tenons à remercier M. Xavier Gélinas d'avoir attiré notre attention sur le lien entre Laurendeau et la franc-maçonnerie.

${ }^{15}$ AÉJ, Archambault à Laurendeau, 20 mars 1907 et Laurendeau à Archambault, 21 mars 1907.

${ }^{10}$ Ibid., Laurendeau à Archambault, 29 août 1907.

${ }^{17}$ Albert Laurendeau, La Vie : considérations biologiques, Saint-Gabriel-de-Brandon, [s.é.], 1911, pp. 169-194.

${ }^{18}$ Ibid., p. 197.

${ }^{19}$ Pour le pape Pie X, le modernisme embrassait toutes les hérésies. (Voir infra, note 20).

${ }^{20}$ Pour un bref exposé des doctrines modernistes, voir Arthur Vermeersch, s.j., «Modernism», The Catholic Encyclopedia, 2e éd., 1913, vol. 10, pp. 415-421.

${ }^{21}$ Albert Laurendeau, La vie..., op. cit., p. 212.

${ }_{22}^{2}$ AÉJ, Archambault à Laurendeau, 16 décembre 1907 et 19 décembre 1907.

${ }^{23}$ AÉJ, Laurendeau à Archambault, 20 décembre 1907.

${ }^{24}$ Agassiz et Quatrefages de Bréau ont rejeté les théories de Lamarck et de Darwin.

${ }^{25}$ AÉJ, Laurendeau à Archambault, 28 décembre 1907 et Archambault à Laurendeau, 30 décembre 1907.

${ }^{26}$ Albert Laurendeau, L'Avenir de la médecine (discours prononcé au Congrès des médecins de langue française à Québec en juillet 1908), p. 8.

${ }^{27}$ Ibid., p. 9.

${ }^{28}$ Ibid., pp. 17, 24.

${ }^{29}$ [S.a., signé «La rédaction»], "Le congrès de Québec», L'Union médicale du Canada, vol. XXXVII, $\mathrm{n}^{\circ} 12$ (décembre 1908), p. 607.

${ }^{30}$ AÉJ, Archambault à Laurendeau, 19 octobre 1910; Laurendeau à Archambault, 20 octobre 1910 et 25 octobre 1910. Nous tenons à remercier le professeur Marcel de Grandpré pour nous avoir fourni le texte du Dr Laurendeau sur L'Avenir de la médecine et pour nous avoir permis de lire son étude inédite sur Laurendeau, «Un moderniste en possession de la vérité». Ce texte nous a aidé à mieux comprendre l'incident de 1910. ${ }^{31}$ Albert laurendeau, La vie..., op. cit., p. 35. Laurendeau prétendrait plus tard qu'il avait voulu écrire «la hiérarchie sociales [AÉ]], Laurendeau à Archambault, 20 juin 1912].

32 Idem, La vie..., op. cit., p. 65.

${ }^{33}$ Ibid., p. 145. Au sujet du transformisme, voir Ernest Mayr, One Long Argument: 
Charles Darvin and the Genesis of Modern Evolutionary Thought, Cambridge, Harvard University Press, 1991, p. 187.

${ }^{34}$ Voir Mayr, op. cit., p. 17, 21, 43; François Jacob, La logique du vivant. Une histoire de l'hérédité, Paris, Gallimard, 1970, pp. 142-152. Au sujet de la science au Québec, voir Luc Chartrand, Raymond Duchesne et Yves Gingras, Histoire des sciences an Québec, Montréal, Boréal, 1987, pp. 159-200; Richard A. Jarrell, «L'ultramontanisme et la science au Canada français", dans Marcel Fournier, Yves Gingras et Othmar Keel, dir. Science et médecine au Québec. Perspectives sociohistoriques, Québec, IQRC, 1987, pp. 41-68; Carl Berger, God and Nature in Victorian Canada, Toronto, UTP, 1983; Peter Eakins and Jean S. Eakins, «Dawson, sir John William» et Jean-Marie Perron, «Provancher, Léon» dans le Dictionnaire biographique du Canada, Tome XII : 1891-1900, Québec, PUL, 1990, pp. 250-258 et 946-948.

${ }^{35}$ Albert Laurendeau, La vie..., op. cit., p. 107. Yvette Conry, Lintroduction du darninisme en France au XIX' siecle, Paris, Vrin, 1974, pp. 308-312. Dans Charles Daruin: The Man and His Influence, Cambridge University Press, 1996, Peter Bowles affirme que "The decades around 1900 saw a growth in the popularity of non-Darwinian mechanisms of evolution and an increased willingness among scientists to proclaim themselves openly hostile to Darvinisms (p. 209). C'est à cette époque que le lamarckisme connut un regain de popularité.

${ }^{36}$ Albert Laurendeau, La vie..., op. cit., p. 97.

${ }^{37}$ Ibid., p. 158.

${ }^{38}$ Ibid, p. 163.

${ }^{39}$ AÉJ, Archambault à Laurendeau, 19 août 1910 et 25 août 1910.

${ }^{40} \mathrm{Joseph}$-Alfred Archambault, "Circulaire de Mgr l'Évêque de Joliette au Clergé de son diocèse. Condamnation du livre La vie : considérations biologiques, L'Étoile du Nord, 28 mars 1912, p. 4. Promulgués par le pape Pie X, le décret Lamentabili sane exitu et l'encyclique Pascendi de 1907 condamnaient les idées modernistes.

${ }^{41}$ AÉj, Laurendeau à Archambault, 20 juin 1912.

${ }^{42}$ AÉJ, Archambault à Laurendeau, 26 juin 1912.

${ }^{43}$ AÉj, Laurendeau à Archambault, 5 mars 1913.

44 [S.a.], «Bulletin religieux», L'Étoile du Nord, 6 mars 1913, p. 6.

${ }^{45}$ AÉJ, Archambault à Laurendeau, 7 mars 1913.

${ }^{46}$ [S.a., signé "Comité de rédaction»], «Albert Laurendeau», La Clinique, vol. XI, n० 6 (septembre 1920), p. 94; [s.a.], «Mort du docteur Alb. Laurendeau», La Presse, 20 août 1920 , p. 10.

${ }^{47}$ Élie J. Auclair, «M $\mathrm{M}^{\mathrm{g}}$ Joseph-Alfred Archambaulb», La Semaine religieuse de Montréal, vol. LXI, $\mathrm{n}^{\circ} 18$ (5 mai 1913), pp. 290-304.

${ }^{48}$ Albert Laurendeau, "Une lettre du docteur Albert Laurendeau, vice-président du Collège des médecins et chirurgiens de la province de Québeor, La Clinique, vol. IX, $\mathrm{n}^{\circ}$ 8 (novembre 1918), p. 257.

${ }^{49}$ Albert Laurendeau, "Une nouvelle lettre du docteur A. Laurendeau», La Clinique, vol. IX, no 9 (décembre 1918), pp. 282-283.

${ }^{50}$ Idem, [s.t.], La Clinique, vol. X, no 1 (avril 1919), p. 27.

${ }^{51}$ Idem, «Le traitement de la grippe», La Clinique, vol. IX, no 10 (janvier 1919), pp. 297 300; «Le traitement de la grippe», LUnion médicale du Canada, vol. XLVIII, ${ }^{\circ} 1$ (janvier 1919), pp. 26-30.

52 [S.a], uLe docteur...), L'Étoile du Nord, 26 août 1920, p. 1.

${ }^{53}$ Typographe, Joseph Guibord était membre de l'Institut canadien de Montréal, même 
à la suite du décret de l'Inquisition (juillet 1869) condamnant ses doctrines libérales et de l'ordonnance de l'évêque de Montréal, $\mathrm{M}^{\mathrm{gr}}$ Ignace Bourget (1799-1885), qui proclamait que «celui qui persiste à vouloir demeurer dans le dit Institut $[\ldots]$ se prive luimême des sacrements à l'heure de la morts (août 1869). Malade, Guibord est décédé trois mois plus tard, toujours membre de l'Institut canadien. Toutefois, il semblerait que l'impénitent Guibord ait reçu l'absolution et la communion peu avant sa mort. Furieux, $\mathrm{M}^{\mathrm{gr}}$ Bourget lui refusa alors une cérémonie religieuse et l'inhumation en terre bénite. Sa veuve, Henriette Brown, avec l'appui des membres de l'Institut canadien, poursuivit alors le curé et les marguilliers de la paroisse Notre-Dame devant les tribunaux dans le but de permettre l'inhumation de son mari dans le cimetière de la Côtedes-Neiges. La cause se rendit jusqu'au Comité judiciaire du Conseil privé de Londres et ne se termina qu'en 1874. C'est alors que le plus haut tribunal de l'Empire ordonna à la paroisse d'inhumer Guibord au cimetière Côte-des-Neiges. La cérémonie se fit sous le regard de la milice canadienne, qu'on a dû mobiliser pour protéger le cortège funèbre. Pour sa part, $\mathrm{M}^{\mathrm{gr}}$ Bourget, déclara le lieu de sépulture sinterdit et séparé du reste du cimetière» en ajoutant que «d̀ repose un révolté que l'on a enterré par la force des armes». Dans l'ensemble, l'affaire Guibord constitue l'affrontement le plus féroce entre le libéralisme radical canadien-français, incarné par l'Institut canadien, et l'école ultramontaine, dont le chef incontesté à l'époque était $\mathrm{M}^{\mathrm{gr}}$ Bourget. (Jean-Roch Rioux, "Guibord, Joseph», dans F. G. Halpenny, dir. Dictionnaire biographique du Canada, Volume IX, Québec, PUL, 1977, pp. 377-378).

${ }^{54}$ Joseph Gauvreau, loc. cit., p. 463.

${ }^{55}$ [Albert Laurendeau], «Intérêt professionneb), La Clinique, vol. I, no 1 (avril 1910), pp. 1-5; «À propos du conseil de discipline», LUnion médicale du Canada, vol. XXXIX, $n^{\circ} 8$ (août 1910), pp. 464-469; A. Lesage, "Réponse de A. Lesage», L'Union médicale du Canada, vol. XXXIX, n 8 (août 1910), pp. 469-470; «Une lettre du docteur Albert Laurendeaum, $L a$ Clinique, vol. V, $n^{\circ} 6$ (septembre 1914), pp. 244-248; [s.a., signé uLa Clinique], «Notre réponse», vol. V, n 6 (septembre 1914), pp. 249-250.

${ }^{56}$ Albert Laurendeau, "Une nouvelle lettre...», loc. cit., p. 283.

${ }^{57}$ Pour une présentation générale de la réception des idées darwinistes au Québec, voir Chartrand, Duchesne et Gingras, op. cit., pp. 179-200.

${ }^{58}$ Cette traduction a été rendue possible grâce à deux fellowships de l'Institut d'études canadiennes de McGill. 